\title{
Usefulness of Narrow-Band Imaging in Endoscopic Submucosal Dissection of the Stomach
}

\author{
Jung-Wook Kim \\ Division of Gastroenterology and Hepatology, Department of Internal Medicine, Kyung Hee University School of Medicine, Seoul, Korea
}

There have been many advances in endoscopic imaging technologies. Magnifying endoscopy with narrow-band imaging is an innovative optical technology that enables the precise discrimination of structural changes on the mucosal surface. Several studies have demonstrated its usefulness and superiority for tumor detection and differential diagnosis in the stomach as compared with conventional endoscopy. Furthermore, magnifying endoscopy with narrow-band imaging has the potential to predict the invasion depth and tumor margins during gastric endoscopic submucosal dissection. Classifications of the findings of magnifying endoscopy with narrow-band imaging based on microvascular and pit patterns have been proposed and have shown excellent correlations with invasion depth confirmed by microscopy. In terms of tumor margin prediction, magnifying endoscopy with narrow-band imaging offers superior delineation of gastric tumor margins compared with traditional chromoendoscopy with indigo carmine. The limitations of narrow-band imaging, such as the need for considerable training, long procedure time, and lack of studies about its usefulness in undifferentiated cancer, should be resolved to confirm its value as a complementary method to endoscopic submucosal dissection. However, the role of magnifying endoscopy with narrow-band imaging is expected to increase steadily with the increasing use of endoscopic submucosal dissection for the treatment of gastric tumors. Clin Endosc 2018;51:527-533

Key Words: Magnifying endoscopy; Narrow band imaging; Stomach; Application; Delineation

\section{INTRODUCTION}

Gastrointestinal endoscopy is an indispensable modality for the diagnosis and treatment of digestive tract diseases. Together with the tremendous increase in its use, the limitations of conventional endoscopy for detecting and discriminating between minute and subtle lesions have been highlighted. Many advances in endoscopic imaging technologies have been made to overcome the shortcomings of conventional endoscopy. The term "image-enhanced endoscopy" (IEE) encompasses various means of improving contrast by using dyes and opti-

Received: October 19, 2018 Accepted: November 1, 2018

Correspondence: Jung-Wook Kim

Division of Gastroenterology and Hepatology, Department of Internal Medicine, Kyung Hee University School of Medicine, 26 Kyungheedae-ro, Dongdaemun-gu, Seoul 02447, Korea

Tel: +82-2-958-8199, Fax: +82-2-968-1848, E-mail: iloveact@hanmail.net ORCID: https://orcid.org/0000-0002-5383-7934

(cc) This is an Open Access article distributed under the terms of the Creative Commons Attribution Non-Commercial License (http://creativecommons.org/ licenses/by-nc/3.0) which permits unrestricted non-commercial use, distribution, and reproduction in any medium, provided the original work is properly cited. cal or electronic techniques. ${ }^{1}$ Among various IEE techniques, chromoendoscopy with dyes has been the traditional and most popular method for detecting lesions or delineating tumor contours since the 1980s, because it enhances mucosal irregularities and surface color differences. ${ }^{2}$ Another novel IEE technique is electronic chromoendoscopy, which includes narrow-band imaging (NBI), i-Scan, and flexible spectral imaging color enhancement. These methods enable digital acquisition of enhanced images through various image-processing techniques, thus eliminating the need for spraying dyes on the mucosa. Among these electronic chromoendoscopy methods, NBI is the most widely applied in clinical practice, and its usefulness has been relatively well demonstrated and validated in many trials. The current clinical application of NBI in the stomach has been expanded to include the detection and diagnosis of various lesions.

Advances in endoscopic devices and techniques have led to many improvements in endoscopic treatment. Endoscopic submucosal dissection (ESD) is preferred for resection of early cancer in the gastrointestinal tract owing to its many 
advantages over endoscopic mucosal resection. ${ }^{3}$ Furthermore, its indications and applicability for use in different organs or diseases have expanded. One of the most important factors affecting clinical outcomes after ESD is the selection of appropriate patients based on the results of careful evaluations. A key point to achieving acceptable patient selection is the determination of invasion depth. During ESD, accurate delineation of the tumor margins is critical for achieving complete resection. For these essential steps, several studies have demonstrated that NBI can be helpful. ${ }^{4-10}$ This review details the clinical application of NBI and focuses on predicting invasion depth and tumor delineation during gastric ESD.

\section{TECHNOLOGICAL BACKGROUND OF NBI}

Since its introduction in 2005, NBI has become one of most the frequently used IEE techniques. ${ }^{11}$ Gono et al. found that the use of 415-nm narrow-band light could improve capillary images, which are obscured under white light. ${ }^{12}$ After Sano reported the potential use of NBI in the gastrointestinal tract, the scope of the application of this technology has considerably expanded. ${ }^{13}$

With the placement of an NBI filter between a xenon lamp and a red-green-blue rotary filter, the spectrum of illumination can be transformed from broad-band blue, green, and red to narrow-band blue $(415 \mathrm{~nm})$ and green $(540 \mathrm{~nm})$ in NBI mode (Fig. 1). ${ }^{11,14}$ This NBI filter is removed under whitelight observation. Although these two narrow-band images (415 and $540 \mathrm{~nm}$ ) are reproduced by an NBI filter, 3 original images must be output to the red, blue, and green channels on the monitor to create a color image. Subsequently, the

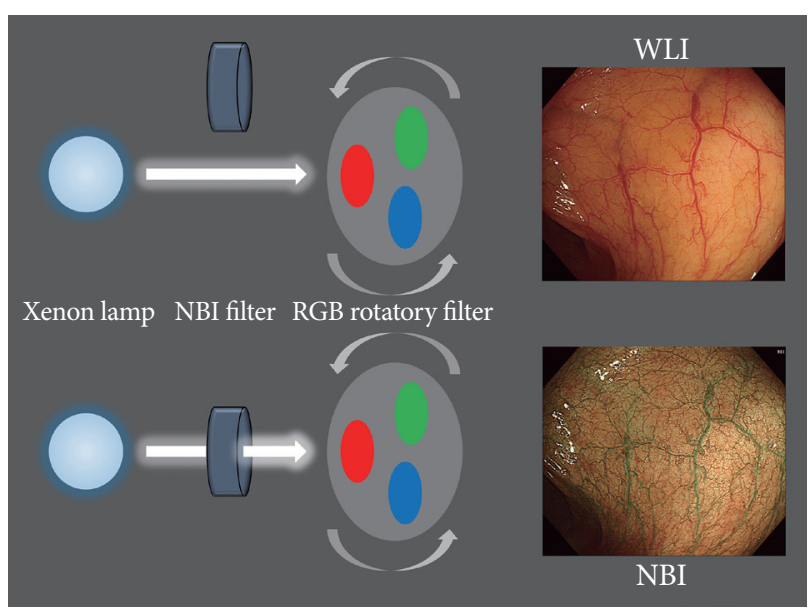

Fig. 1. Schema of the narrow-band imaging (NBI) system. RGB, red-greenblue; WLI, white-light image.
$415 \mathrm{~nm}$ image is allocated to the blue and green channels so that blood vessels on the superficial mucosal layer are reproduced in brown, whereas the $540 \mathrm{~nm}$ image is allocated to the red channel so that the vessels in the deeper layer can be expressed as greenish-blue. ${ }^{14}$ The thin capillary network on the mucosal surface is brownish, whereas thick blood vessels are cyan. Blood vessels are revealed clearly because hemoglobin is a chromophore that determines the color of the mucosa. The 2 narrow-band illuminations used in the NBI system, 415 and $540 \mathrm{~nm}$, coincide with the 2 absorption peaks of hemoglobin. Therefore, blue narrow-band illumination has strong scattering and absorption properties that can enhance the visualization of blood vessels. ${ }^{11}$

\section{MAGNIFYING ENDOSCOPY WITH NBI}

Several studies have demonstrated that the findings of magnification endoscopy had excellent correlation with histological diagnoses in premalignant gastric lesions and early gastric cancer (EGC). ${ }^{15-18}$ Magnifying endoscopy combined with NBI (M-NBI) can maximize the usefulness of NBI in various organs, especially the stomach. NBI images without magnification are too dark to identify the structural and color changes in organs with a large lumen such as the stomach. ${ }^{14} \mathrm{~A}$ dark image is seen because the NBI system uses only 2 narrow illumination lights, whereas conventional white-light imaging uses wide-band visible light. ${ }^{14}$

In clinical practice, high-definition magnifying endoscopes (GIF-H260Z and GIF-H290Z; Olympus Co., Tokyo, Japan) and an electronic endoscopy system (EVIS LUCERA ELITE VIDEO SYSTEM; Olympus Co.) are commonly used to observe gastric lesions. The structure enhancement function of the video system is usually set at a level of 8 for magnifying observation. ${ }^{19}$ A black soft hood mounted at the tip of the scope is essential to optimize examinations. It enables endoscopists to maintain a distance of about $2 \mathrm{~mm}$ between the tip of the zoom lens and the mucosal surface. ${ }^{20}$ Clear and maximally magnified images can be obtained using this detailed manipulation technique.

\section{CLINICAL APPLICATION OF NBI IN THE STOMACH}

As mentioned above, NBI is only applicable in the stomach when it is used with magnifying endoscopy. It enhances the mucosal microvascular architecture and surface pattern to detect faint mucosal changes that occur in response to various conditions in the stomach. Many studies have reported its 
feasibility for identifying subtle changes associated with atrophy, intestinal metaplasia, Helicobacter pylori infection, and EGC. ${ }^{12,15-18,21-24}$ Among these, its role in discriminating EGC, which is of considerable concern to endoscopists, has been studied widely. ${ }^{6,1621-23,25}$ Focusing on EGC, several studies have shown that M-NBI is useful in the detection of tumors and for prediction of tumor differentiation, invasion depth, and margins. ${ }^{4-8,10,16,21-27}$

Muto et al. suggested the diagnostic criteria for EGC when using M-NBI. ${ }^{25}$ The results of their study showed excellent accuracy with use of minimal criteria consisting of the triad of disappearance of fine mucosal structure, microvascular dilatation, and microvascular pattern heterogeneity. ${ }^{24} \mathrm{An}$ other Japanese study group proposed endoscopic diagnostic criteria for small and depressed EGC when using M-NBI, based on a previous report that used magnifying endoscopy alone. ${ }^{23,28}$ They defined 2 findings seen in M-NBI, as follows: (1) a demarcation line (DL) between the cancerous lesion and the surrounding noncancerous area and (2) an irregular microvascular pattern inside the lesion. An analysis of 40 EGC cases found that M-NBI in addition to conventional whitelight endoscopy had 96.6\% accuracy, 95.0\% sensitivity, and $96.8 \%$ specificity. ${ }^{23}$ Recently, the Japanese Gastroenterological Association and its associated society established a working group, and proposed a unified algorithm for the classification of the diagnosis of EGC. ${ }^{25}$ In this simple diagnostic algorithm, if a suspicious lesion is detected, the identification of a DL is the first step. If a DL is identified, the presence of an irregular microvascular and/or microsurface pattern should be determined in the next step. If both conditions are met, the diagnosis of EGC can be made (Fig. 2). ${ }^{25}$

Some studies have investigated the prediction of EGC differentiation by using magnifying endoscopy alone and with M-NBI. ${ }^{22,28}$ In differentiated adenocarcinoma cases, findings related to the microvascular architecture include the

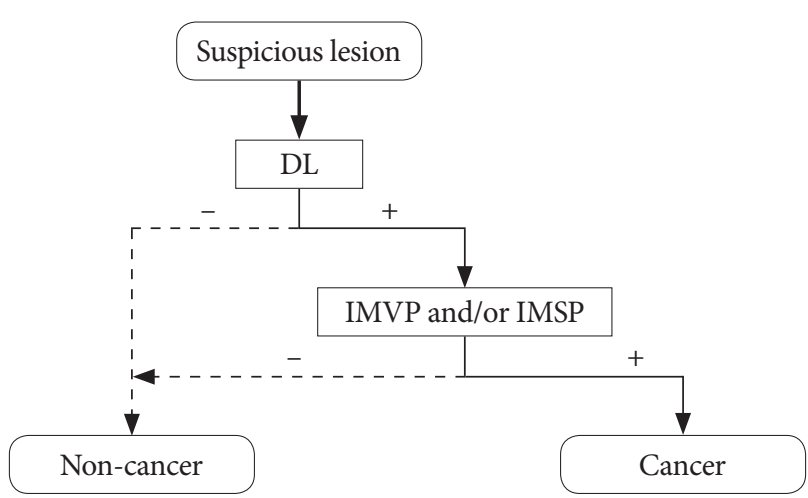

Fig. 2. Flow diagram of the simple diagnostic algorithm for magnifying endoscopy in gastric cancer proposed by Muto et al. ${ }^{25} \mathrm{DL}$, demarcation line; IMVP, irregular microvascular pattern; IMSP, irregular microsurface pattern. disappearance of the regular subepithelial capillary network pattern, presence of an irregular microvascular pattern, and presence of a DL. In contrast, an ill-defined area with reduced microvascular pattern was observed in undifferentiated adenocarcinoma. ${ }^{28}$ Nakayoshi et al. attempted to predict the histological characteristics of gastric cancer by using M-NBI. ${ }^{22}$ They classified the microvascular structures observed with M-NBI into a relatively regular fine network and an irregular corkscrew capillary pattern. ${ }^{22}$ The former pattern was highly correlated with differentiated cancer and the latter was highly correlated with undifferentiated cancer $(66.1 \%$ and $85.7 \%$, respectively).

Although the results from several studies showed an excellent correlation between M-NBI findings and the diagnosis and differentiation of EGC, they should be confirmed with histopathologic examinations before ESD. In other words, M-NBI alone is insufficient to definitively diagnose EGC and indicate ESD without histologic confirmation. However, the invasion depth and tumor margins can be confirmed only after ESD. Therefore, it is likely that M-NBI will play an important role in making decisions about ESD in terms of predicting the invasion depth and tumor margins. These issues will be discussed in the following sections.

\section{PREDICTION OF INVASION DEPTH}

The determination of invasion depth plays a critical role in establishing the treatment strategy for EGC, such as ESD or surgery. Conventional endoscopy and endoscopic ultrasonography (EUS) are the mainstay techniques for evaluating invasion depth in EGC. Some studies showed that the overall accuracy of conventional endoscopy for assessing invasion depth was $75 \%-82 \%{ }^{26,27,29}$ Tsujii et al. demonstrated that EUS may have an additional benefit of the endoscopic prediction of invasion depth in EGC. ${ }^{29}$ They suggested that an integrated diagnostic strategy combining conventional endoscopy and EUS had a comprehensive accuracy of $>85 \%{ }^{29}$ In contrast, another study showed that EUS had lower accuracy than conventional endoscopy $(67.4 \%$ vs. $73.7 \%, p<0.001){ }^{26}$ This may mean that about $20 \%$ of patients with EGC cannot receive optimal treatment after careful evaluation with only conventional endoscopy and EUS. Owing to the need for a novel modality to overcome this limitation, some researchers have investigated the feasibility of M-NBI for predicting invasion depth in cases of EGC.

Yagi et al. categorized magnified NBI images of differentiated EGC cases based on the microvascular and pit pattern into mesh, loop, and interrupted patterns. ${ }^{21}$ They found an excellent correlation between M-NBI and invasive change; $94.9 \%$ of 
patients with a mesh or loop pattern had mucosal cancer and 92.3\% with an interrupted pattern had submucosal (SM) cancer. ${ }^{21}$ Another group tried to discriminate SM2 cancer from mucosal and SM1 cancer by using M-NBI because SM2 cancer requires surgical resection. ${ }^{10}$ They showed that the M-NBI findings of unstructured, scattered, and multicaliber vessels had the potential to indicate SM2 invasion in cases of differentiated EGC. ${ }^{10}$ Kikuchi et al. suggested that invasion depth could be predicted more simply by the presence or absence of dilated vessels. ${ }^{7}$ The diagnostic criterion of the presence of dilated vessels alone showed a diagnostic accuracy, sensitivity, and specificity of $81.5 \%, 37.5 \%$, and $88.3 \%$, respectively. Although some studies have demonstrated the potential of M-NBI for predicting invasion depth in EGC, an established classification system and well-designed studies are needed to enable its application in routine clinical practice.

\section{DETERMINING TUMOR MARGINS}

Compared with endoscopic mucosal resection, one of the main advantages of ESD is that it can be used to resect tumors regardless of size. However, this is only possible if the tumor margins can be clearly identified. Endoscopists sometimes encounter obscure lateral margins in EGC owing to gastritis and/or intestinal metaplasia in the mucosa surrounding the tumor. Traditionally, conventional white-light endoscopy and/ or chromoendoscopy with indigo carmine have been used to determine gastric tumor margins (Fig. 3). Several studies have demonstrated that the accuracy rate of chromoendos- copy with indigo carmine for predicting tumor margins was $75.9 \%-85.7 \% .4,5,8,30$ In other words, about $20 \%$ of patients who underwent ESD are at risk of incomplete resection due to inaccurate delineation.

M-NBI is currently the most promising method for determining tumor margins and characterizing lesions. ${ }^{31}$ Its findings are used in the detection of EGC features such as a DL and irregularity of surface and microvascular patterns (Fig. 4). M-NBI is relatively well established as a tool for delineating EGC cases. ${ }^{6,20,23}$ Several prospective and retrospective studies have demonstrated that M-NBI offers superior delineation of gastric tumor margins compared with chromoendoscopy with indigo carmine. ${ }^{4,89}$ A randomized trial from Japan compared M-NBI and chromoendoscopy with indigo carmine for determining tumor margins in 118 gastric tumor cases. ${ }^{9}$ The accuracy of tumor margin marking was significantly higher in the M-NBI group than in the chromoendoscopy group (97.4\% vs. $77.8 \%, p=0.009$ ). Another recent prospective study also showed the excellent accuracy of M-NBI for evaluating the horizontal extent of EGC compared with chromoendoscopy (89.4\% vs. $75.9 \%, p=0.0071){ }^{4} \mathrm{M}-\mathrm{NBI}$ successfully delineated all margins in $72.6 \%$ of cases, even in those with an unclear margin on chromoendoscopy, in a case series of 350 patients with EGC. ${ }^{8}$

\section{DUAL-FOCUS MAGNIFICATION WITH UPDATED NBI}

As mentioned earlier, NBI should be combined with mag-
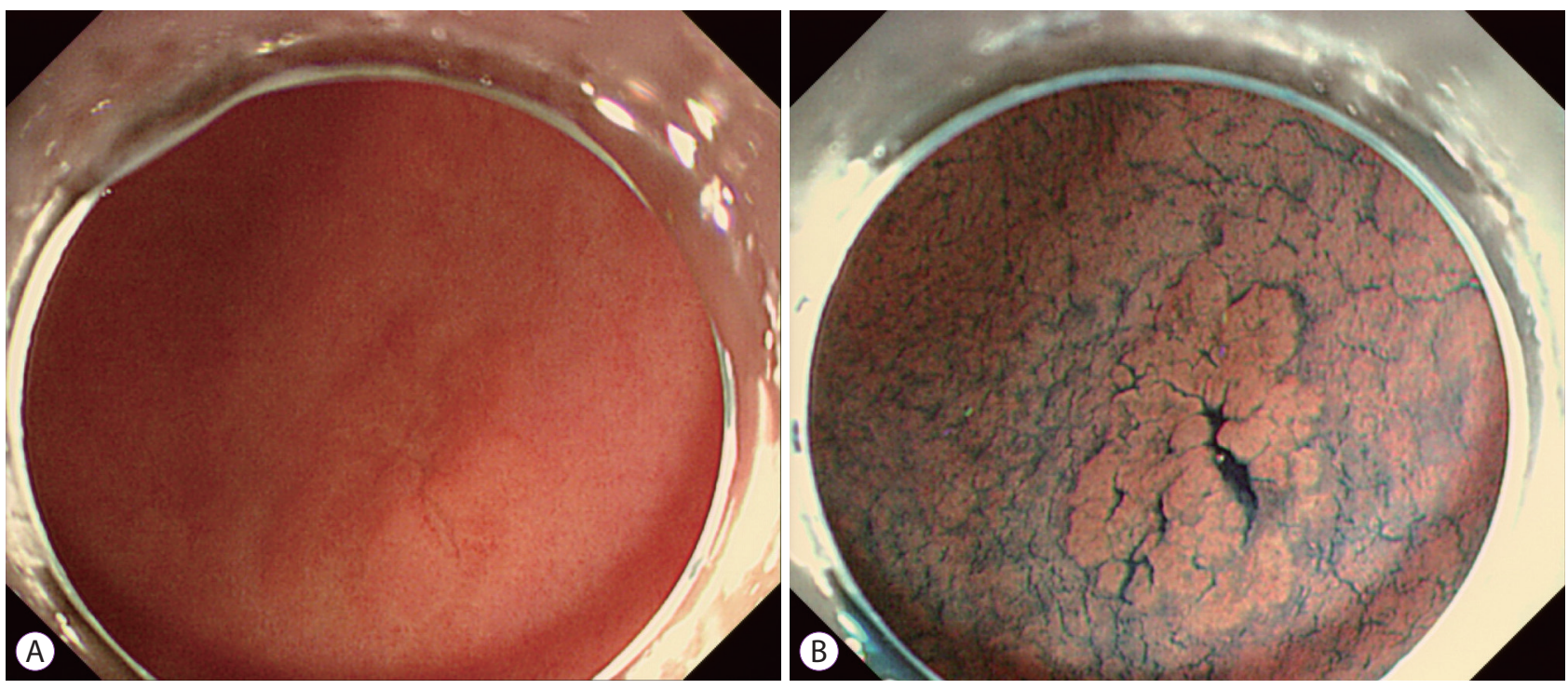

Fig. 3. Chromoendoscopic finding of gastric adenoma with low-grade dysplasia. (A) A conventional white-light image showing an unclear lesion with ambiguous margins. (B) After indigo carmine spraying, a slightly elevated lesion with a clear margin is evident. 
nifying endoscopy in the stomach because of the noise and darkness. A newly developed dual-focus (DF) endoscope (GIF-HQ290; Olympus Co.) and updated NBI system (EVIS LUCERA ELITE, CV-190; Olympus Co.) have addressed these limitations of conventional NBI systems. Along with increased brightness of the updated NBI system, simplified digital magnification involving the push of a button was adopted (Fig. 5A). ${ }^{32}$ The DF function has a novel optical innovation that allows the endoscopist to select between a normal mode and a near-focus mode. The near-focus mode is optimized for near-field observation with 45-fold magnification (Fig. 5B). ${ }^{32,33}$
Some studies have addressed the usefulness of the updated NBI system. ${ }^{32-36}$ Goda et al. identified the noninferiority of DF-NBI for detecting superficial carcinoma in the pharynx and esophagus compared with conventional M-NBI. ${ }^{31}$ Another Japanese study group reported that DF-NBI was also useful for the differential diagnosis of diminutive colorectal lesions. ${ }^{36}$ Although a retrospective study from the United Kingdom demonstrated that DF-NBI improved the diagnostic yield of upper gastrointestinal mucosal lesions, its usefulness for gastric lesions is questionable. ${ }^{37}$ Further studies are needed to confirm whether this updated NBI system can replace the
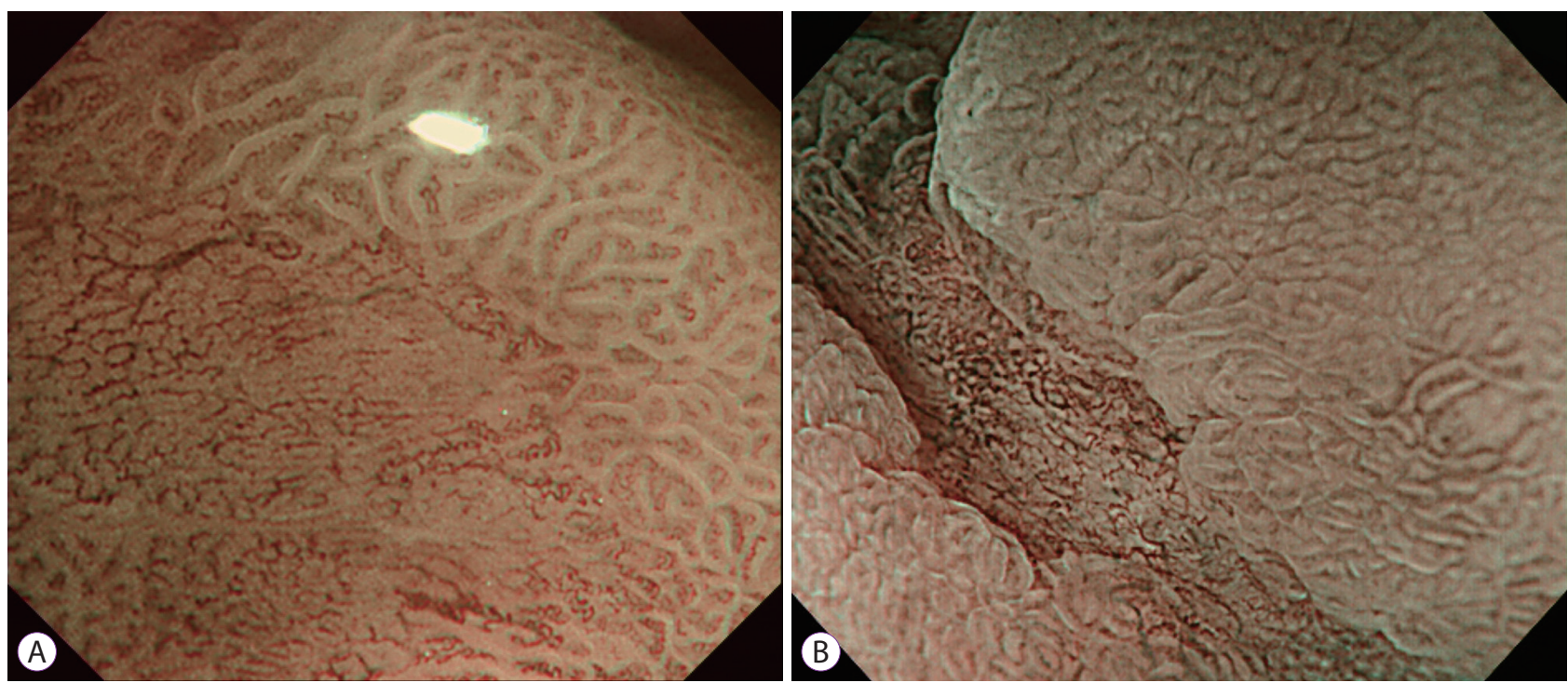

Fig. 4. Early gastric cancer observed using magnifying endoscopy with narrow-band imaging. (A) A demarcation line is clearly revealed. (B) The tumor margins are well delineated based on the irregular microvascular pattern.
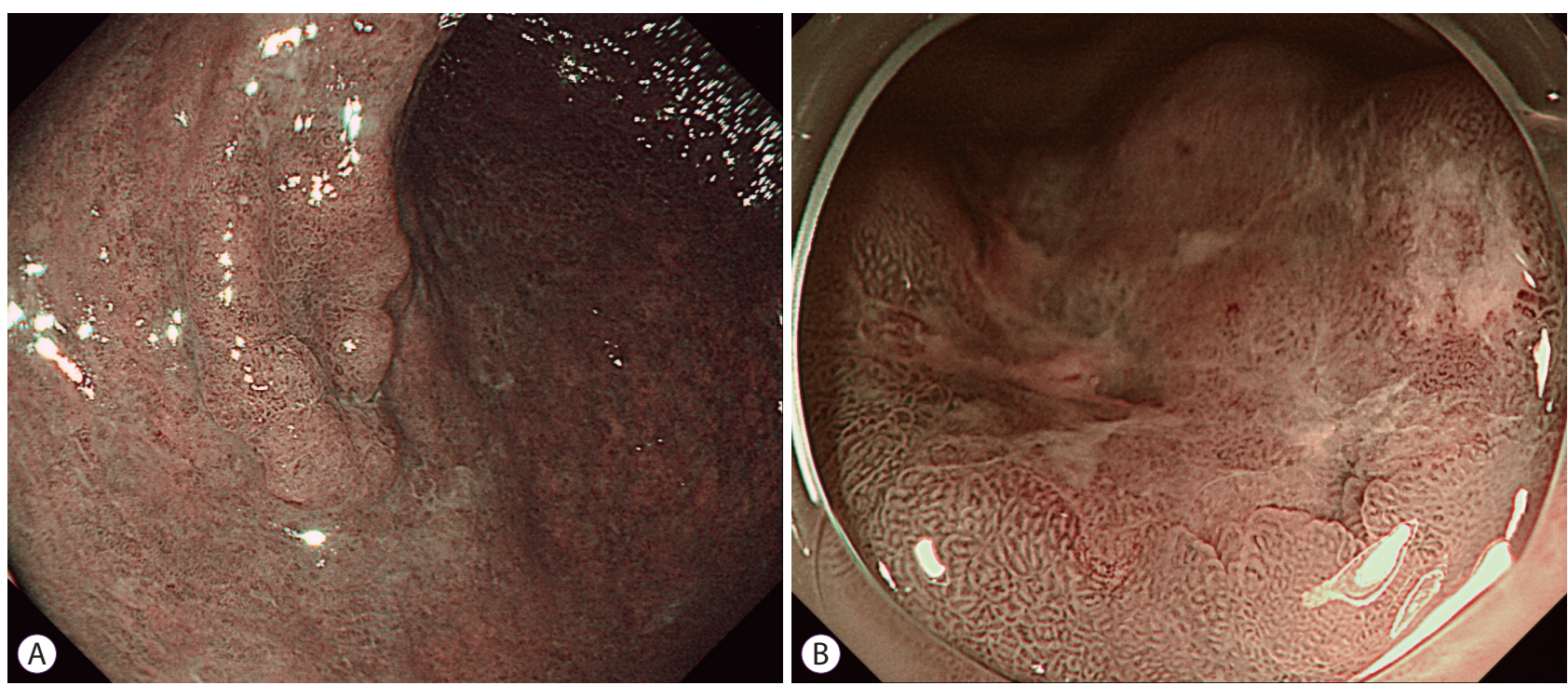

Fig. 5. Dual-focus magnification with the updated narrow-band imaging (NBI) system. (A) Updated NBI system with an improved light source that allows brighter images. (B) The near-focus mode of dual-focus NBI provides accurate delineation of the tumor by about 45 -fold near-field magnification. 
conventional M-NBI system.

\section{LIMITATIONS OF NBI}

Despite its considerable potential for clinical application in the stomach, NBI has some limitations that need to be overcome in the ESD era. In terms of the procedure, endoscopists should be fully trained and should have gained considerable experience to ensure optimal M-NBI performance. Delineation before ESD can be time consuming, especially in cases of large tumors. Its role in predicting invasion depth and tumor margin in EGC is limited to differentiated cancers. Despite studies identifying the feasibility of M-NBI for accurate delineation, it is challenging to determine tumor margins in undifferentiated cancers. ${ }^{4,8,9}$ Furthermore, few studies evaluating the usefulness of predicting invasion depth have been performed in cases of differentiated cancer. This is probably because undifferentiated cancer often spreads within the lamina propria sparsely before its exposure on the mucosal surface. ${ }^{38}$ Another reason is that even patients with small undifferentiated cancers are more likely to undergo surgery than ESD. Therefore, M-NBI may not have a critical role for the detection of undifferentiated cancers at present. The expansion of the indications of ESD as a treatment for diminutive undifferentiated cancers will increase the need for M-NBI. It is also expected that technological advances such as DF-NBI will compensate for these limitations and increase the utilization of NBI in the stomach.

\section{CONCLUSIONS}

In the ESD era, the usefulness of IEE techniques such as $\mathrm{M}-\mathrm{NBI}$ is expanding based on the results from several studies. M-NBI is an innovative optical technology that enables precise discrimination of the morphological changes on the gastric mucosal surface. It could be an especially useful modality during ESD in terms of predicting invasion depth and enabling accurate delineation of tumor margins. Limitations such as the requirement for considerable training, long procedure time, and the lack of studies about its usefulness in undifferentiated cancer should be resolved if $\mathrm{M}-\mathrm{NBI}$ is to become a popular and attractive complementary method to ESD. The role of M-NBI is expected to expand together with the increasing use of ESD in the treatment of gastric tumors. Future well-designed studies are also needed to confirm the effect of M-NBI on the outcomes of ESD.
Conflicts of Interest

The author has no financial conflicts of interest.

\section{Acknowledgments}

The author greatly appreciates Prof. Jae-Young Jang from Kyung Hee University School of Medicine, Seoul, Korea, for providing high-quality images.

\section{REFERENCES}

1. Jang JY. The past, present, and future of image-enhanced endoscopy. Clin Endosc 2015;48:466-475.

2. Kudo S, Hirota S, Nakajima T, et al. Colorectal tumours and pit pattern. J Clin Pathol 1994;47:880-885.

3. Oka S, Tanaka S, Kaneko I, et al. Advantage of endoscopic submucosal dissection compared with EMR for early gastric cancer. Gastrointest Endosc 2006;64:877-883.

4. Asada-Hirayama I, Kodashima S, Sakaguchi Y, et al. Magnifying endoscopy with narrow-band imaging is more accurate for determination of horizontal extent of early gastric cancers than chromoendoscopy. Endosc Int Open 2016;4:E690-E698.

5. Nagahama T, Yao K, Uedo N, et al. Delineation of the extent of early gastric cancer by magnifying narrow-band imaging and chromoendoscopy: a multicenter randomized controlled trial. Endoscopy 2018;50:566-576.

6. Yao K, Anagnostopoulos GK, Ragunath K. Magnifying endoscopy for diagnosing and delineating early gastric cancer. Endoscopy 2009;41:462467.

7. Kikuchi D, lizuka T, Hoteya S, et al. Usefulness of magnifying endoscopy with narrow-band imaging for determining tumor invasion depth in early gastric cancer. Gastroenterol Res Pract 2013;2013:217695.

8. Nagahama T, Yao K, Maki S, et al. Usefulness of magnifying endoscopy with narrow-band imaging for determining the horizontal extent of early gastric cancer when there is an unclear margin by chromoendoscopy (with video). Gastrointest Endosc 2011;74:1259-1267.

9. Kiyotoki S, Nishikawa J, Satake M, et al. Usefulness of magnifying endoscopy with narrow-band imaging for determining gastric tumor margin. J Gastroenterol Hepatol 2010;25:1636-1641.

10. Kobara H, Mori H, Fujihara S, et al. Prediction of invasion depth for submucosal differentiated gastric cancer by magnifying endoscopy with narrow-band imaging. Oncol Rep 2012;28:841-847.

11. Gono K. Narrow band imaging: technology basis and research and development history. Clin Endosc 2015;48:476-480.

12. Gono K, Obi T, Yamaguchi M, et al. Appearance of enhanced tissue features in narrow-band endoscopic imaging. J Biomed Opt 2004;9:568577.

13. Sano Y. New diagnostic method based on color imaging using narrow-band imaging (NBI) system for gastrointestinal tract. Gastrointest Endosc 2001;53:AB125.

14. Muto M, Horimatsu T, Ezoe Y, Morita S, Miyamoto S. Improving visualization techniques by narrow band imaging and magnification endoscopy. J Gastroenterol Hepatol 2009;24:1333-1346.

15. Nakagawa S, Kato M, Shimizu Y, et al. Relationship between histopathologic gastritis and mucosal microvascularity: observations with magnifying endoscopy. Gastrointest Endosc 2003;58:71-75.

16. Tajiri H, Doi T, Endo H, et al. Routine endoscopy using a magnifying endoscope for gastric cancer diagnosis. Endoscopy 2002;34:772-777.

17. Kim S, Harum K, Ito M, Tanaka S, Yoshihara M, Chayama K. Magnifying gastroendoscopy for diagnosis of histologic gastritis in the gastric antrum. Dig Liver Dis 2004;36:286-291.

18. Guelrud M, Herrera I, Essenfeld H, Castro J, Antonioli DA. Intestinal 
metaplasia of the gastric cardia: a prospective study with enhanced magnification endoscopy. Am J Gastroenterol 2002;97:584-589.

19. Yao K, Takaki Y, Matsui T, et al. Clinical application of magnification endoscopy and narrow-band imaging in the upper gastrointestinal tract: new imaging techniques for detecting and characterizing gastrointestinal neoplasia. Gastrointest Endosc Clin N Am 2008;18:415-433, vii-viii.

20. Yao K. Clinical application of magnifying endoscopy with narrow-band imaging in the stomach. Clin Endosc 2015;48:481-490.

21. Yagi K, Nakamura A, Sekine A, Umezu H. Magnifying endoscopy with narrow band imaging for early differentiated gastric adenocarcinoma. Dig Endosc 2008;20:115-122.

22. Nakayoshi T, Tajiri H, Matsuda K, Kaise M, Ikegami M, Sasaki H. Magnifying endoscopy combined with narrow band imaging system for early gastric cancer: correlation of vascular pattern with histopathology (including video). Endoscopy 2004;36:1080-1084.

23. Ezoe $\mathrm{Y}$, Muto M, Uedo N, et al. Magnifying narrowband imaging is more accurate than conventional white-light imaging in diagnosis of gastric mucosal cancer. Gastroenterology 2011;141:2017-2025.e3.

24. Kato M, Kaise M, Yonezawa J, et al. Magnifying endoscopy with narrow-band imaging achieves superior accuracy in the differential diagnosis of superficial gastric lesions identified with white-light endoscopy: a prospective study. Gastrointest Endosc 2010;72:523-529.

25. Muto M, Yao K, Kaise M, et al. Magnifying endoscopy simple diagnostic algorithm for early gastric cancer (MESDA-G). Dig Endosc 2016;28:379393.

26. Choi J, Kim SG, Im JP, Kim JS, Jung HC, Song IS. Comparison of endoscopic ultrasonography and conventional endoscopy for prediction of depth of tumor invasion in early gastric cancer. Endoscopy 2010;42:705713 .

27. Choi J, Kim SG, Im JP, Kim JS, Jung HC, Song IS. Endoscopic prediction of tumor invasion depth in early gastric cancer. Gastrointest Endosc 2011;73:917-927.

28. Yao K, Oishi T, Matsui T, Yao T, Iwashita A. Novel magnified endoscopic findings of microvascular architecture in intramucosal gastric cancer.
Gastrointest Endosc 2002;56:279-284.

29. Tsujii Y, Kato M, Inoue T, et al. Integrated diagnostic strategy for the invasion depth of early gastric cancer by conventional endoscopy and EUS. Gastrointest Endosc 2015;82:452-459.

30. Iizuka T, Kikuchi D, Hoteya S, Yahagi N. The acetic acid + indigocarmine method in the delineation of gastric cancer. J Gastroenterol Hepatol 2008;23:1358-1361.

31. Goda K, Dobashi A, Yoshimura N, et al. Dual-focus versus conventional magnification endoscopy for the diagnosis of superficial squamous neoplasms in the pharynx and esophagus: a randomized trial. Endoscopy 2016;48:321-329.

32. Goda K, Dobashi A, Tajiri H. Perspectives on narrow-band imaging endoscopy for superficial squamous neoplasms of the orohypopharynx and esophagus. Dig Endosc 2014;26 Suppl 1:1-11.

33. Singh R, Jayanna M, Navadgi S, Ruszkiewicz A, Saito Y, Uedo N. Narrow-band imaging with dual focus magnification in differentiating colorectal neoplasia. Dig Endosc 2013;25 Suppl 2:16-20.

34. Singh R, Shahzad MA, Tam W, et al. Preliminary feasibility study using a novel narrow-band imaging system with dual focus magnification capability in Barrett's esophagus: is the time ripe to abandon random biopsies? Dig Endosc 2013;25 Suppl 2:151-156.

35. Wallace MB, Crook JE, Coe S, et al. Accuracy of in vivo colorectal polyp discrimination by using dual-focus high-definition narrow-band imaging colonoscopy. Gastrointest Endosc 2014;80:1072-1087.

36. Ikematsu $H$, Matsuda $T$, Osera $S$, et al. Usefulness of narrow-band imaging with dual-focus magnification for differential diagnosis of small colorectal polyps. Surg Endosc 2015;29:844-850.

37. Bond A, Burkitt MD, Cox T, et al. Dual-focus magnification, high-definition endoscopy improves pathology detection in direct-to-test diagnostic upper gastrointestinal endoscopy. J Gastrointestin Liver Dis 2017;26:19-24.

38. Li HY, Ge ZZ, Fujishiro M, Li XB. Current clinical applications of magnifying endoscopy with narrow band imaging in the stomach. Diagn Ther Endosc 2012;2012:271914. 